\section{Does the traditional snakebite severity score correctly classify envenomated patients?}

\author{
Seungho Kang, Jeongmi Moon, Byeongjo Chun \\ Department of Emergency Medicine, Chonnam National University Hospital, Chonnam National University \\ Medical School, Gwangju, Korea
}

Objective This study aims to help set domestic guidelines for administration of antivenom to envenomated patients after snakebites.

Methods This retrospective observational case series comprised 128 patients with snake envenomation. The patients were divided into two groups according to the need for additional antivenom after the initial treatment based on the traditional snakebite severity grading scale. One group successfully recovered after the initial treatment and did not need any additional antivenom $(n=85)$ and the other needed an additional administration of antivenom $(n=43)$.

Results The group requiring additional administration of antivenom showed a higher local effect score and a traditional snakebite severity grade at presentation, a shorter prothrombin and activated partial prothrombin time, a higher frequency of rhabdomyolysis and disseminated intravascular coagulopathy, and longer hospitalization than the group that did not need additional antivenom. The most common cause for additional administration was the progression of local symptoms. The independent factor that was associated with the need for additional antivenom was the local effect pain score (odds ratio, 2.477; 95\% confidence interval, 1.309 to 4.689). The optimal cut-off value of the local effect pain score was 1.5 with $62.8 \%$ sensitivity and $71.8 \%$ specificity.

Conclusion When treating patients who are envenomated by a snake, and when using the traditional snakebite severity scale, the local effect pain score should be taken into account. If the score is more than 2, additional antivenom should be considered and the patient should be frequently assessed.

Keywords Snakes; Venoms; Antivenins
eISSN: 2383-4625

Received: 25 December 2015

Revised: 26 January 2016

Accepted: 26 January 2016

Correspondence to: Jeongmi Moon Department of Emergency Medicine, Chonnam National University Hospital, Chonnam National University Medical School, 42 Jebong-ro, Dong-gu, Gwangju 61469, Korea

E-mail:jmmoon@chonnam.ac.kr

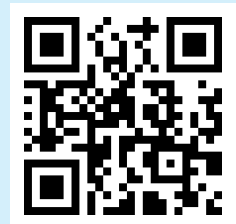

How to cite this article:

Kang S, Moon J, Chun B. Does the traditional snakebite severity score correctly classify envenomated patients? Clin Exp Emerg Med 2016;3(1):34-40.

This is an Open Access article distributed under the terms of the Creative Commons Attribution Non-Commercial License (http:// creativecommons.org/licenses/by-nc/3.0/). 


\section{INTRODUCTION}

There are about 600 species of poisonous snakes, and four of them inhabit Korea. Gloydius brevicaudus, Gloydius ussuriensis, and Gloydius intermedius belong to the Viperidae family, and Rhabdophis tigrinus belongs to the Cloubridae family. ${ }^{1,2}$ When a person is bitten by a poisonous snake, venom is injected, causing localized symptoms of mild pain and edema and generalized ones including dyspnea, ptosis, mental alteration, and tachycardia. In severe cases, patients develop acute renal failure, myocardial infarction, disseminated intravascular coagulation, and even death. ${ }^{1,3}$ Treatment for poisonous snakebite is divided into supportive care and antivenom administration. Most studies regarding snake antivenom focus on the Gloydius brevicaudus (Stejneger) family from northern America. As a result, the textbooks recommend antivenom doses with North American standards, which are significantly greater than the current practice in Korea (4 to 6 vials of antivenom during the initial state). ${ }^{4,5}$ There is no standardized guideline on antivenom administration in Korea except for some research papers. ${ }^{6.7}$

The ideal way to estimate the appropriate dose of antivenom is to measure the exact amount of venom delivered, which is not possible. Instead, guidelines suggest predicting the amount based on severity of symptoms at presentation. However, it is very difficult to predict the expansion of localized and systematic symptoms. In some cases, generalized symptoms develop in the absence of localized symptoms. When venom spreads slowly via the lymphatics and veins, delayed systemic symptoms may develop after localized ones. ${ }^{4,8}$ Additional administration of antivenom after the initial dose is required in many cases.

To be effective, antivenom should be administered within a certain time window. When the window is missed, the dose should be increased. ${ }^{19,10}$ When the initial dose is not sufficient, it may result in worse clinical outcomes. ${ }^{6}$

In this study, the authors aim to identify factors associated with re-administration of antivenom for patients who initially received antivenom based on the traditional snakebite severity score.

\section{METHODS}

\section{Study subjects}

Patients over 18 years old who visited the study site from January 2005 to May 2014 with a poisonous snakebite within 24 hours were included. A case of poisonous snakebite was defined when the shape of the poisonous snake was witnessed by the patients or the wound was consistent with snake fangs. "Cases were excluded if antivenom had been administered not according to protocol, if antivenom was pre-administered at another hospital before the patient was transferred to the study site, if the time of the snakebite was not clear, if the patient was pregnant, if the patient left the study site against medical advice, or if the patient was transferred to another facility before treatment was completed.

The snakebite protocol includes proper dressing, tetanus immunization, pain control, and elevation of the wounded part. The protocol also includes categorizing the snakebite severity with the traditional snakebite severity scale (TSSS) with a grade of 0 to 4. For grade 0, no antivenom is suggested. For grades 1 and 2 , one vial $(6,000$ units) of antivenom is recommended. For grade 3 , 12,000 units are recommended, and for grade 4, 18,000 units. For all cases, antivenom Agkistrodon halys (Kovax, Seoul, Korea) was used. After the decision for antivenom administration was made, antihistamine and the selected dose of antivenom were mixed in $100 \mathrm{~mL}$ of normal saline. The solution was given to patients intravenously at a rate of $1 \mathrm{~mL} / \mathrm{min}$ for 15 minutes, which was increased to $100 \mathrm{~mL} / \mathrm{hr}$ after assurance of no adverse effect. The initial administration of antivenom was carried out immediately after patients' presentation. Additional administration was only decided by emergency physicians. The decision was made based on the presence of local edema, pain, worsening signs, newly appearing general symptoms, and abnormal coagulation tests. General symptoms include altered mental status, diplopia, blurred vision, and dyspnea.

\section{Measures and outcomes}

Subjects were divided into two groups according to whether additional antivenom was administered. When only initial treatment according to TSSS was necessary, patients were grouped as the initial treatment (IT) group. When additional treatment was required, they were grouped as the additional treatment (AT) group.

A retrospective chart review was conducted to identify potential factors predicting case severity. Factors considered included age, sex, comorbidities, height, weight, wound location, treatment prior to visit, snakebite severity, interval to antivenom administration, presenting vital signs, and laboratory findings. Wound location was classified as distal when they were on the fingers or toes, and the rest were defined as proximal. ${ }^{12}$ The severity of snakebite was evaluated based on TSSS and the local effect index of Scharmanrhk Noffsinger. ${ }^{13}$ We compared the outcomes between the two groups, which included adverse effects of antivenom, complications during hospital stay, length of hospital stay, and mortality. Complications included rhabdomyolysis, acute kidney injury, disseminated intravascular coagulopathy (DIC), and any conditions that were considered to be associated with the snakebite. Rhabdomyolysis was defined as a creatine kinase 
level higher than five times the normal range. DIC was defined as a condition with elevation of prothrombin time (PT), activated partial prothrombin time (aPTT), D-dimer, fibrinogen degradation products, and decreased platelet and fibrinogen levels. ${ }^{1,7}$ Acute kidney injury was defined as an increase of creatinine by $0.3 \mathrm{mg} /$ $\mathrm{dL}$ or $50 \%$ from initial findings. Liver injury was defined as an increase of alanine transaminase by more than $100 \mathrm{U} / \mathrm{L}^{14,15} \mathrm{We}$ collected data on timing, dose, and indications for additional administration of antivenom.

\section{Statistical analysis}

Continuous variables were described as mean \pm standard deviation, or median along with the interquartile range. Categorical variables were described with frequencies. We used the Shapiro Wilk test to test whether continuous variables were consistent with a normal distribution. When a variable followed a normal distribution, an independent t-test or analysis of variance was used. When a variable did not follow a normal distribution, a Mann-Whitney U-test, or Kruskal-Wallis test was used. To ana- lyze categorical variables, a chi-square test was used. We performed a multivariate analysis using potentially significant variables identified by univariate analysis. Odds ratios, 95\% confidence intervals, and P-values were calculated by the multivariate analysis. The identified factor that was significant from the multivariate analysis was re-analyzed to determine a receiver operating characteristics curve, area under the curve, and Youden index to determine the best cut-off value. We used IBM SPSS ver. 21.0 (IBM Corp., Armonk, NY, USA), and only P-values below 0.05 were considered significant.

This study was conducted at the Chonnam National University Hospital, and approved by the Chonnam National University Hospital institutional review board.

\section{RESULTS}

\section{Participants' basic characteristics}

Overall, 128 patients were included for the study among 267 patients who were treated for poisonous snakebite. Patients were

Table 1. Baseline characteristics of envenomated patients

\begin{tabular}{|c|c|c|c|c|}
\hline Variable & $\begin{array}{c}\text { Total } \\
(n=128)\end{array}$ & $\begin{array}{l}\text { Single administration } \\
\qquad(n=85)\end{array}$ & $\begin{array}{l}\text { Additional adminstration } \\
\qquad(n=43)\end{array}$ & P-value \\
\hline Age (yr) & $57.7 \pm 20.7$ & $56.8 \pm 22.1$ & $59.5 \pm 17.6$ & 0.485 \\
\hline Male & $79(61.7)$ & $52(61.2)$ & $27(62.8)$ & 0.859 \\
\hline Body mass index $\left(\mathrm{kg} / \mathrm{m}^{2}\right)$ & $22.8 \pm 3.4$ & $22.6 \pm 4.0$ & $23.1 \pm 2.3$ & 0.540 \\
\hline \multicolumn{5}{|l|}{ Underlying disease } \\
\hline Hypertension & $13(10.2)$ & $9(10.7)$ & $4(9.3)$ & 0.804 \\
\hline Diabetes mellitus & $8(6.3)$ & $6(7.1)$ & $2(4.7)$ & 0.584 \\
\hline Systolic blood pressure $(\mathrm{mmHg})$ & $129.4 \pm 24.9$ & $127.5 \pm 27.4$ & $133.2 \pm 18.7$ & 0.224 \\
\hline Heart rate (/min) & $78.3 \pm 11.1$ & $78.21 \pm 0.9$ & $78.51 \pm 1.4$ & 0.816 \\
\hline \multicolumn{5}{|l|}{ Bite site } \\
\hline Digital & $60(46.9)$ & $42(49.4)$ & $18(41.9)$ & 0.419 \\
\hline Prehospital procedure & & & & 0.759 \\
\hline Incision and suction & $13(10.2)$ & $8(9.4)$ & $5(11.6)$ & \\
\hline Constriction band & $5(3.9)$ & $4(4.7)$ & $1(2.3)$ & \\
\hline Time interval from bite to antivenin adminstration & & & & 0.722 \\
\hline Interval $\leq 2 \mathrm{hr}$ & $60(46.9)$ & $38(44.7)$ & $22(51.2)$ & \\
\hline $2 \mathrm{hr}<$ interval $\leq 4 \mathrm{hr}$ & $17(13.3)$ & $11(12.9)$ & $6(14.0)$ & \\
\hline $4 \mathrm{hr}<$ interval $\leq 6 \mathrm{hr}$ & $15(11.7)$ & $9(10.6)$ & $6(14.0)$ & \\
\hline $6 \mathrm{hr}<$ interval $\leq 8 \mathrm{hr}$ & $11(8.6)$ & $9(10.6)$ & $2(4.7)$ & \\
\hline $8 \mathrm{hr}<$ interval $<24 \mathrm{hr}$ & $25(19.5)$ & $18(21.2)$ & $7(16.3)$ & \\
\hline \multicolumn{5}{|l|}{ Local effect score } \\
\hline Pain & $1.4 \pm 0.8$ & $1.2 \pm 0.7$ & $1.8 \pm 0.9$ & $<0.001$ \\
\hline Swelling & $1.6 \pm 0.9$ & $1.3 \pm 0.7$ & $2.1 \pm 0.9$ & $<0.001$ \\
\hline Ecchymosis & $0.3 \pm 0.7$ & $0.2 \pm 0.6$ & $0.4 \pm 0.8$ & 0.105 \\
\hline Time & $0.6 \pm 0.6$ & $0.4 \pm 0.6$ & $0.8 \pm 0.5$ & $<0.001$ \\
\hline Traditional snake bite scale score & & & & $<0.001$ \\
\hline Grade 0 & $1(0.8)$ & $1(1.2)$ & $0(0.0)$ & \\
\hline Grade I & $87(68.0)$ & 69 (81.2) & $18(41.9)$ & \\
\hline Grade II & $39(30.5)$ & $15(17.6)$ & $24(55.8)$ & \\
\hline Grade III & $1(0.8)$ & $0(0.0)$ & $1(2.3)$ & \\
\hline
\end{tabular}

Values are presented as mean \pm standard deviation or number (\%). 
Table 2. Initial laboratory findings of envenomated patients

\begin{tabular}{lcccc}
\hline Variable & $\begin{array}{c}\text { Total } \\
(\mathrm{n}=128)\end{array}$ & $\begin{array}{c}\text { Single administration } \\
(\mathrm{n}=85)\end{array}$ & $\begin{array}{c}\text { Additional adminstration } \\
(\mathrm{n}=43)\end{array}$ & P-value \\
\hline $\mathrm{pH}$ & $7.42 \pm 0.04$ & $7.42 \pm 0.03$ & $7.42 \pm 0.04$ & 0.780 \\
$\mathrm{HCO}_{3}{ }^{-}(\mathrm{mmol} / \mathrm{L})$ & $23.4 \pm 3.6$ & $23.3 \pm 4.0$ & $23.7 \pm 2.7$ & 0.540 \\
$\mathrm{WBC}\left(\mathrm{mm}^{3}\right)$ & $9,150.0 \pm 4,201.6$ & $8,951.7 \pm 3,951.4$ & $9,542.0 \pm 4,681.3$ & 0.455 \\
Platelets $\left(\times 10^{3} / \mathrm{mm}^{3}\right)$ & $220.3 \pm 68.9$ & $226.7 \pm 68.0$ & $207.8 \pm 69.9$ & 0.144 \\
AST (U/L) & $28.0(23.3-83.8)$ & $26.5(22.3-50.0)$ & $38.5(28.8-146.8)$ & 0.275 \\
ALT (U/L) & $26.0(14.8-48.5)$ & $24.0(14.0-47.0)$ & $32.5(19.3-48.5)$ & 0.779 \\
BUN (mg/dL) & $16.4 \pm 5.7$ & $16.1 \pm 5.4$ & $17.0 \pm 6.0$ & 0.407 \\
Cr (mg/dL) & $0.8 \pm 0.3$ & $0.8 \pm 0.2$ & $0.8 \pm 0.3$ & 0.094 \\
Creatinine kinase (U/L) & $201.0(124.0-442.0)$ & $181.0(18.3-291.0)$ & $290.0(127.0-1,406.0)$ & 0.059 \\
CK-MB (ng/mL) & $7.1(3.1-18.0)$ & $7.2(3.1-13.1)$ & $7.1(2.6-35.7)$ & 0.803 \\
Myoglobin (ng/mL) & $56.0(34.3-239.3)$ & $68.5(34.3-239.3)$ & $45.5(30.8-601.8)$ & 0.603 \\
Troponin I (ng/mL) & $0.01(0.01-0.02)$ & $0.01(0.01-0.02)$ & $0.01(0.01-0.08)$ & 0.304 \\
PT (INR) & $1.0 \pm 0.3$ & $1.0 \pm 0.3$ & $0.9 \pm 0.3$ & 0.013 \\
aPTT (sec) & $32.3 \pm 9.5$ & $33.9 \pm 7.1$ & $29.2 \pm 12.5$ & 0.028 \\
Fibrinogen (mg/dL) & $231.2(195.1-272.2)$ & $234.5(205.4-272.9)$ & $223.7(176.6-268.8)$ & 0.651 \\
\hline
\end{tabular}

Values are presented as mean \pm standard deviation or median (interquartile range).

WBC, white blood cell; AST, aspartate aminotransferase; ALT, alanine aminotransferase; BUN, blood urea nitrogen; $\mathrm{Cr}$, creatinine; CK-MB, creatine kinase MB; PT, prothrombin time; INR, International normalized ratio; aPT, activated partial prothrombin time.

Table 3. Clinical course of envenomated patients

\begin{tabular}{lcccc}
\hline Variable & $\begin{array}{c}\text { Total } \\
(\mathrm{n}=128)\end{array}$ & $\begin{array}{c}\text { Single } \\
\text { administra- } \\
\text { tion }(\mathrm{n}=85)\end{array}$ & $\begin{array}{c}\text { Additional } \\
\text { administra- } \\
\text { tion }(\mathrm{n}=43)\end{array}$ & P-value \\
$\begin{array}{l}\text { Side effect related with } \\
\text { antivenom }\end{array}$ & 0 & 0 & 0 & \\
$\begin{array}{l}\text { Systemic sign } \\
\text { Dizziness }\end{array}$ & $14(10.9)$ & $9(10.6)$ & $5(11.6)$ & 0.859 \\
$\quad$ Blurred vision & $4(3.1)$ & 0 & $4(9.3)$ & 0.148 \\
Dyspnea & $3(2.3)$ & $1(1.2)$ & $2(4.7)$ & 0.220 \\
Diplopia & $2(1.6)$ & $1(1.2)$ & $1(2.3)$ & 0.621 \\
Ptosis & $1(0.8)$ & 0 & $1(1.2)$ & 0.475 \\
$\quad$ Mental change & $1(0.8)$ & 0 & $1(2.3)$ & 0.158 \\
Complications & & & & \\
$\quad$ Rhabdomyolysis & $32(25.0)$ & $16(18.9)$ & $16(37.2)$ & 0.028 \\
Skin defect & $12(9.4)$ & $5(5.9)$ & $7(16.3)$ & 0.057 \\
Acute liver injury & $9(7.0)$ & $6(7.1)$ & $3(7.0)$ & 0.981 \\
DIC & $7(5.5)$ & 0 & $7(16.3)$ & $<0.001$ \\
Acute kidney injury & $2(1.6)$ & $1(1.2)$ & $1(2.3)$ & 0.627 \\
Hospitalization (day) & $5.7 \pm 7.0$ & $4.6 \pm 8.1$ & $7.9 \pm 3.1$ & 0.011 \\
\hline
\end{tabular}

Values are presented as number (\%) or mean \pm standard deviation.

DIC, disseminated intravascular coagulation.

categorized into two groups: 85 in the IT group, 43 in the AT group. The average age was 57.7 years, and 78 (61.7\%) were men (Table 1). An average of 6.8 hours was measured from the time of bite to administration of antivenom. For $46.9 \%$ of cases, the wound was on distal parts, such as the toes or fingers.

Additional treatment was not associated with age, the interval before treatment, or location of wound. The AT group showed a significantly higher rate of grade II, and a higher local effect in- dex of pain, edema, and time. The IT group showed an elevated value of PT and aPTT (Table 2).

\section{Comparison of clinical progress}

No adverse event was reported from either group (Table 3). Generalized symptoms during the hospital stay included dizziness (10.9\%), blurred vision (3.1\%), and dyspnea (2.3\%), without a significant difference between the two groups (Table 3). Complications included rhabdomyolysis (25.0\%), and two of these patients developed acute kidney injury afterward. DIC occurred in seven patients (5.5\%), without clinically significant bleeding. Incidences of rhabdomyolysis and DIC were significantly higher for the AT group (Table 3). The hospital length of stay was 7.9 days in the IT group, which was significantly higher than the 4.6 days in the AT group. All subjects were discharged from hospital without significant comorbidities.

\section{Information regarding additional administration of antivenom}

Eighteen patients (20.7\%), 24 (61.5\%), and one (100\%) of grades $\mathrm{I}, \mathrm{II}$, and III, respectively, received an additional administration of antivenom. The overall number of re-administrations was 76 . The median amount of antivenom was one, two, and four vials for grades II II, and III, respectively. There was no statistically significant difference in the amount among these grades (Table 4). The average interval between antivenom administrations was 9.0 hours. There was no significant difference in the interval between the 
Table 4. Information related with administered antivenom

\begin{tabular}{|c|c|c|c|c|}
\hline Variable & Grade I $(n=18)$ & Grade II $(n=24)$ & Grade III $(n=1)$ & P-value \\
\hline No. of additional administrations & $1.0(1.0-2.0)$ & $2.0(1.0-2.0)$ & 2 & 0.295 \\
\hline \multicolumn{5}{|l|}{ Antivenom (vials) } \\
\hline Total amount during hospitalization & $2.0(2.0-3.0)$ & $3.0(2.0-3.0)$ & 6 & 0.092 \\
\hline \multicolumn{5}{|l|}{ Time interval $\left.(\mathrm{hr})^{\mathrm{a}}\right)$} \\
\hline To second administration & $8.0(4.0-26.3)$ & $7.5(4.0-22.5)$ & 12 & 0.911 \\
\hline
\end{tabular}

Values are presented as median (interquartile range).

a) Time interval from first administration of antivenom.

Table 5. Multiple regression analysis for independent factors associated with the need of additional administration of antivenom

\begin{tabular}{lccc}
\hline Variable & Odd ratios & P-value & $\begin{array}{c}95 \% \text { confidence } \\
\text { interval }\end{array}$ \\
\hline Local effect score-pain & 2.477 & 0.005 & $1.309-4.689$ \\
\hline
\end{tabular}

first and the last administration among the three grades. The indications for additional treatment were worsening of local symptoms (72.1\%), abnormal coagulation panel (25.3\%), and appearance of new generalized symptoms $(2.3 \%)$.

\section{Initial findings associated with additional treatment}

The univariate analysis revealed that among initial presentations, the local pain effect index, PT, aPT, and TSSS were significantly associated with outcome. The local swelling effect index was removed since it is already included in the TSSS. The time local effect index was also removed from the final analysis because it can only be measured 8 hours after the snakebite. The multivariate analysis using these variables found that the pain local effect index was a significant predictor of need for additional antivenom (Table 5).

The receiver operating characteristics curve found an area under the curve of pain local effect index of 0.704 . The cut off value with the best Youden index was 1.5, with a sensitivity of $62.8 \%$ and specificity of $71.8 \%$.

\section{DISCUSSION}

In this study, when the TSSS was used to determine initial dose of antivenom administration, $33.6 \%$ of patients required additional doses. Another study with 62 patients with grade 0 to grade II envenomation also found $32.1 \%$ required additional antivenom. ${ }^{6}$

Proper initial dosing is critical for poisonous snakebite's clinical course. ${ }^{6}$ This study found the AT group had a longer hospital stay, and a higher rate of rhabdomyolysis and DIC. The reasons for additional doses may be insufficient initial dose or a proper dose with excessive speed of excretion compared to that of the toxin.
However, the form of antitoxin in Korea is immunoglobulin $\mathrm{G}$ antibody, which has a half-life of 61 to 194 hours, ${ }^{2,5}$ which is much longer than the time interval of 9 hours before additional administration in this study. This implies that additional administration is more likely due to insufficient initial dose rather than excessive excretion.

In this study, the major reasons for additional administration were primarily local symptoms rather than generalized symptoms, and the last administration of antivenom was 28 hours after the initial one, indicating that the severity of symptoms changed over time. Initial dosage was largely based on edema and generalized symptoms, and the fact that dosage was determined at the very first stage of disease progression may be the reason for the need for additional dosing. Moreover, the dosage protocol is based on research in the US, which may not be fully applicable to Korean circumstances.

In this study, age, body mass index, and interval to antivenom administration were not associated with additional administration. This may be explained by the fact that the severity of snakebite is determined by several factors including the patients' underlying conditions, body volume, interval for administration, type and amount of toxin, and depth of initial wound. Even for a certain species of snake, the toxic severity differs with size, age, and season. ${ }^{4}$

Snake venom is a mixture of enzymes, polypeptides, and nontoxic proteins. These components influence coagulation pathways independently or as a group, in both accelerating and decelerating ways. ${ }^{2.4}$ In this study, the IT group showed longer aPTT and PT than the AT group. This is not consistent with the expectation that the AT group may have longer aPTT and PT, and it requires further research on the association between severity and changing coagulopathy. Still, it is very difficult to use this knowledge in clinical settings since the laboratory results are not consistent with outcome.

The TSSS grade was associated with increased likelihood of additional administration of antivenom. For grade I and II, additional administration was necessary for $20.7 \%$ and $61.5 \%$, respec- 
tively. Another study with 32 patients and an initial dose of two vials showed the progression rates for grades I and II envenomation were $38.5 \%$ and $60.0 \%$, respectively. ${ }^{16}$

Our multivariate analysis showed that presenting pain local effect index was an independent factor predicting additional antivenom administration. Pain is a local symptom like edema, which occurs immediately after the bite, significantly sooner than generalized symptoms, which occur after the toxin enters the circulatory system. Considering that the progression of local symptoms and signs is the most frequent reason for additional administration, taking both local and generalized symptoms into account will enhance the accuracy of the TSSS.

Adverse events related to antivenom include urticaria, rash, anaphylaxis, and delayed onset of serum sickness. In the US, acute events affect $5.4 \%$ to $14.3 \%$ of patients, and $16 \%$ develop serum sickness. ${ }^{12,17}$ However, we did not see adverse reactions associated with antivenom, which is consistent with other studies in Korea, which have reported very low rates from 0.5 to $0.8 \%{ }^{16,18}$ This discrepancy may be explained by different dosing strategies and antivenom production protocols.

This study has some limitations. First, the venom differs among species of snakes, which will influence the outcome. ${ }^{2}$ Although specific treatment for each species may be helpful for improving outcomes, it is extremely rare that patients can accurately identify the snake, which is a major limitation.

Second, there are regional differences of snake inhabitation, which may influence the symptom presentations. Since this study was performed in a single center, regional specifics of the area may have influenced the outcome.

Third, this study is based on a retrospective chart review, which did not include information on the quality of prehospital treatment. However, prehospital treatments are only associated with skin necrosis. They do not influence factors associated with initial administration dosing strategy, such as diplopia, headache, and coagulopathy. ${ }^{18}$

Fourth, the pain local effect index is influenced by analgesics administered prior to the visit. Since the protocol for snakebite does not include a pain control strategy, need for pain relief was determined on a clinical basis. This may have influenced the selection of the initial dose. A chart review has revealed that in most cases, acetaminophen and non-steroidal anti-inflammatory drugs were used except for 3.2\% of cases when an opioid was used.

Last, we only included a very small number of grade 0 and III patients. This may be because only severely affected patients visit tertiary centers, and about $75.5 \%$ of snakebite victims in Korea have stage I and II envenomation. ${ }^{2}$

In conclusion, we recommend measuring the pain local effect index along with TSSS when deciding upon administration of antivenom. Specially, when the pain index is above two, additional administration is highly likely and repeated evaluation of the patient is required. A further study to confirm these findings is necessary.

\section{CONFLICT OF INTEREST}

No potential conflict of interest relevant to this article was reported.

\section{REFERENCES}

1. Jin SC, Lee JW, Yang SJ, Joo MD, Choi WI. Consideration of factors associated with complications and systemic symptoms of snake bites. J Korean Soc Emerg Med 2008;19:686-96.

2. Lim H, Kang HG, Kim KH. Antivenom for snake bite in Korea. J Korean Med Assoc 2013;56:1091-103.

3. Mahmood K, Naqvi IH, Talib A, et al. Clinical course and outcome of snake envenomation at a hospital in Karachi. Singapore Med J 2010;51:300-5.

4. Nelson L, Lewin N, Howland MA, Hoffman R, Goldfrank L, Flomenbaum N. Goldfrank's toxicologic emergencies. 9th ed. San Francisco, CA: McGraw Hill; 2011.

5. Auerbach PS. Wilderness medicine. 6th ed. Philadelphia, PA: Elsevier; 2012.

6. You KM, Kwon WY, Kwon TH, Shin JH, Lee HJ. Optimal dose of antivenin for asymptomatic or minor envenomation patient with korean viperidae injuries. J Korean Soc Emerg Med 2013; 24:420-7.

7. Han SK, Kim IS, Ryu S, et al. The effectiveness of antivenin in treating snake bites resulting in minimal clinical symptoms. J Korean Soc Emerg Med 2007;18:577-83.

8. Hurlbut KM, Dart RC, Spaite D, McNally J. Reliability of clinical presentation for predicting significant pit viper envenomation. Ann Emerg Med 1988;17:438-9.

9. Nam SH, Lee HW, Ryu BY, Kim HK, Souk H, Choi CS. The evaluation of severe complications in snake bite. J Korean Surg Soc 1995;49:856-66

10. Spiller HA, Bosse GM, Ryan ML. Use of antivenom for snakebites reported to United States poison centers. Am J Emerg Med 2010;28:780-5.

11. Paik NK, Sim JH. Snakes. Seoul: Isungsa; 1999.

12. Dart RC, McNally J. Efficacy, safety, and use of snake antivenoms in the United States. Ann Emerg Med 2001;37:181-8.

13. Scharman EJ, Noffsinger VD. Copperhead snakebites: clinical severity of local effects. Ann Emerg Med 2001;38:55-61. 
14. Moon JM, Chun BJ, Lee BK. Glasgow coma scale score in the prognosis of acute carbamate insecticide intoxication. Clin Toxicol (Phila) 2012;50:832-7.

15. Moon JM, Chun BJ. Acute endosulfan poisoning: a retrospective study. Hum Exp Toxicol 2009;28:309-16.

16. Kim DH, Choe SM, Oh YM, Oh JS, Kyong YY, Choi KH. Clinical significance of delayed re-evaluation in initial symptoms following snakebite injury. J Korean Soc Clin Toxicol 2009;7:97-
104.

17. Cannon R, Ruha AM, Kashani J. Acute hypersensitivity reactions associated with administration of crotalidae polyvalent immune Fab antivenom. Ann Emerg Med 2008;51:407-11.

18. Jeon JC, Lee DH, Kwon GY, Kim SJ. Relation of first aid associated with complications after snake bites. J Korean Soc Clin Toxicol 2009;7:105-112. 